\title{
THREE-DIMENSIONAL QUANTITATIVE STRUCTURE-ACTIVITY RELATIONSHIPS MODELING STUDIES OF PHYTOCHEMICALS FROM BRASSICACEAE AS POTENT INHIBITORS AGAINST TUMOR INFLAMMATION
}

\author{
Renukadevi J ${ }^{1 *}$, Nandhinidevi $G^{1}$, Bavanilatha $\mathbf{M}^{2}$, Kavitha Bharathy $\mathbf{T}^{\mathbf{1}}$, Jameela Banu $\mathbf{M}^{1}$, Kanimozhi $\mathbf{S}^{1}$ \\ ${ }^{1}$ Department of Biotechnology, Anna University, Chennai - 600 025, Tamil Nadu, India. ${ }^{2}$ Department of Biotechnology, Sathyabama \\ University, Chennai - 600 119, Tamil Nadu, India. Email: neelarenu@gmail.com
}

Received: 24 September 2016, Revised and Accepted: 10 October 2016

\begin{abstract}
Objective: The aim of this study was to develop a three-dimensional quantitative structure-activity relationships (3D-QSARs) model for studying the interaction of different phytochemicals with nuclear factor kappa B (NF-kB) inducing kinase, a major regulator in tumor inflammation.

Methods: Different phytochemicals (ligands) from Brassicaceae were selected and tested for Lipinski's rule of five and further analyzed using interaction studies (docking) to identify the binding site in the target protein. Ligands with best fit were made to pass through ADMET filter, and the nontoxic ligands were selected based on the pIC50 values.
\end{abstract}

Results: The 3D-QSARs of the ligands were designed using comparative molecular field analysis, and glucoraphanin was found to be stable and fit after subjecting for molecular dynamics simulation with annealing studies.

Conclusion: Thus, the model may be prospectively used in drug design to find possible inhibitors of NF-kB, which plays a key prominent role in cancer inflammation.

Keywords: Three-dimensional quantitative structure-activity relationships, Brassica oleracea, Simulation, Annealing, Nuclear factor kappa B kinase.

(C) 2017 The Authors. Published by Innovare Academic Sciences Pvt Ltd. This is an open access article under the CC BY license (http://creativecommons. org/licenses/by/4. 0/) DOI: http://dx.doi.org/10.22159/ajpcr.2017.v10i1.15361

\section{INTRODUCTION}

Inflammation is a physiological process that can eliminate the agent responsible for cell injury/tissue damage and initiate tissue repair by launching a well-coordinated immune response. It is a protective response involves complex molecular mechanism involving various cytokines and cell mediators [1,2]. Chronic inflammation leads to increased production of macrophages which results in abnormal growth of cells leads to tumor. Toll-like receptors (TLRs) activate inflammatory signals when foreign body enters the cell. These TLRs detect pathogen-derived products and couple to different adapter proteins which activate respective transcription factors such as nuclear factor kappa B (NF-kB). These transcription factors induce the release of various immune and inflammatory cytokines such as tumor necrosis factor and interleukin (IL)-6, which proved to be excellent targets for inflammatory diseases. These receptors activate the cells firstresponder mechanism, which turns on major transcription factors that quickly activate genes to prepare cells for the damage. These factors are proving to be essential in certain kinds of cancers. NF-kB is already present in cells, which is held in an inactive state by a protein known as $I \kappa B \alpha[3,4]$. By adding phosphate group to $\mathrm{I} \kappa \mathrm{B} \alpha$, it gets neutralized. NF- $\mathrm{KB}$ is now free to move into the nucleus of the cell and activate a wide range of genes that promote cell growth, survival, migration, and the angiogenesis. In the context of cancer, the activation of NF-kB in immune cells in turn induces molecules that attract more immune cells into the tumor [3]. It is a feedforward loop that results in an uncontrolled growth, with the immune cells actively enhancing the Hallmark capabilities of the cancer cells. Red cabbage contains glucosinolates such as glucoraphanin, glucoiberin, and gluconapin, which are under basic research for their potential properties of affecting some types of cancer [4,5,6]. Phytochemicals from Brassicaceae such as glucosinolates and its breakdown products such as sulforaphane and Indole 3 carbinol were found to have potential effects over mediators which promote inflammation 3 [7]. Glucosinolates are hydrolyzed to isothiocyanates (ITCs) by the action of myrosinase. ITCs, possibly a bioactive component in cruciferous vegetables, are being investigated for their chemopreventive and chemotherapeutic effects [8-10]. Quantitative structure-activity relationships (QSARs) have been applied for decades in the development of relationships between physicochemical properties of chemical substances and their biological activities to obtain a reliable statistical model for prediction of the activities of new chemical entities [11-13]. The fundamental principle is that the difference in structural properties is responsible for the variations in biological activities of the compounds. In this study QSAR model was designed based on COMFA analysis, it will define the structural descriptors essential for antiinflammatory action based on NF-kB kinase. The results will provide the toxicity range and will aid in the development of lead against inflammation related complications.

\section{METHODS}

Structure preparation

A total of 28 different ligands of Brassicaceae were retrieved from PUBCHEM and those structures were saved in sdf format. Their geometry was stabilized. The structure of receptor NF-kB inducing kinase) structure was retrieved from PDB and saved in pdb file format. The PDB ID of this receptor is 4G3D. Drug likeness of the ligands was assessed using Lipinski's rule of five using Pre-ADMET.

\section{Interaction studies}

The interaction studies of the selected ligands with the NF-kB are studied by flexible docking using accelrys discovery studio (ADS). Water molecules and other ligands attached to the protein molecule were removed, and force field was applied and checked for receptor cavities and active-binding site in the receptor molecule. The active sites were changed manually for every ligand. After the successful completion of docking, the 
dock score was used to find the best interaction. Dock score=Ligand/ receptor interaction energy+ligand internal energy Complex with dock score of above 40 was considered as having good interactions. Suitable ligands were filtered on the basis of docking results $[14,15]$.

\section{Pharmacokinetics-toxicity}

ADME-Tox refers to absorption, distribution, metabolism, and excretiontoxicity in pharmacokinetics study profile of drug. ADMET was done for the 14 ligands which had the best dock score using ADMET descriptor in ADS. The ADMET descriptor protocol estimates a range of ADMET-related properties for small molecules such as solubility level, blood brain barrier level, CYP450 2D6, Hepatotoxicity, and plasma-binding level. After the ADMET prediction, 7 ligands were sorted out from 14 ligands as non-toxic and reported as flavanol, glucoiberin, gluconapin, glucoraphanin, neochlorogenic acid, phenylethyl isothiocyanate, and progoitrin (Table 1).

\section{Three-dimensional (3D)-QSAR modeling}

3D-QSAR analysis was performed using the commercial software SYBYL. It uses comparative molecular field analysis (CoMFA) technique which is based on data from known active molecules. To apply CoMFA, activities and 3D structure of molecules are needed. The aim of CoMFA is to derive a correlation between the biological activity of a set of molecules and their 3D shape, electrostatic, and hydrogen bonding characteristics. It involves the sequential steps which includes screening molecules with the same set of interaction followed by optimizing the molecular mechanics. Alignment of different models will generate a suitable model with best fit based on the quantum mechanic equation. Molecules with activities spanning about three log units of ki or IC50 values are required. In this study, we have considered pIC50 values of the non-toxic 7 ligands for CoMFA analysis.

The CoMFA model depends on the molecules alignment and errors in this step can provide 3D QSAR models that have a low predictive power. In order to compute the value of the steric field on a certain grid point $j$, the steric probe is placed in that grid point and the van der Waals interaction energy is calculated. The electrostatic field values in each grid point are computed by placing a positively or negatively charged atom in the grid point:

$$
\mathrm{E}_{\mathrm{c}, j}=\sum_{j=1}^{n} \frac{q_{i} q_{j}}{\varepsilon r_{i j}}
$$

where $\mathrm{E}_{\mathrm{c}, j}$ is the value of the electrostatic field at the grid point $j, \mathrm{q}_{i}$ is the partial charge of atom $i$ in the molecule, $\mathrm{q}_{j}$ is the charge of the probe atom located at the grid point $j$, and $\varepsilon$ is the dielectric constant. The grid points inside the van der Waals molecular surface were excluded from the calculation of the electrostatic field. The values of the steric and electrostatic fields are collected into a QSAR table in order to be correlated with the biological activity values.

The pIC50 values of the various ligands were selected from literature [16]. IC50 refers to the half maximal inhibitory concentration which is a measure of the effectiveness of a substance in inhibiting a specific biological or biochemical function. IC50 values were converted using an online tool for predicting pIC50 values from IC50 values.

\section{Molecular dynamics simulation}

Molecular dynamics simulation is a computational method that calculates the time-dependent behavior of a molecular system. Molecular dynamics simulations provide detailed information on the fluctuations and conformational changes of proteins and nucleic acids. These methods are used to investigate the structure, dynamics, and thermodynamics of biological molecules and their complexes. They are also used in the determination of structures from X-ray crystallography and from nuclear magnetic resonance experiments. The structure of glucoraphanin with target protein NF-kB was to be analyzed, and this interactive region in target protein may play a role in stabilization of the rest of the protein residues. To predict the stability of active compound, molecular dynamics simulation was produced for 1000 ns of the active compound glucoraphanin with NF-kB protein. The dynamics was then analyzed, and the interacting residues were found.

\section{Simulated annealing}

Simulated annealing is a type of molecular dynamics experiment, in which the temperature of the model of glucoraphanin with NF-kB protein is cycled over time with widely conformational space. The usual reasons to invoke this method are to obtain several different lowenergy conformations of a single molecule or to obtain several different low-energy configurations of a system of molecules. Here, simulated annealing applies a high temperature of $500 \mathrm{~K}$ for 10 cycles.

\section{RESULTS AND DISCUSSION}

\section{Interaction studies}

Fourteen ligands had good interaction with NF-kB protein. The interaction was defined based on the dock score of the ligand and the receptor. Ligands with dock score of 40 and above were selected for further rounds of the study. The top 4 interacted ligands are given in Fig. 1 along with their dock score.

\section{D-QSAR interpretation}

The pIC50 values were taken as activity data for the compounds. The predicted activities of the compounds were derived by the COMFA model. The top QSAR model was observed to be "glucoraphanin" with a predicted pIC50 value of $4.28 \mu \mathrm{m}$. The measured and the predicted values are given in Fig. 2.

The yellow and green colors represent the steric activity of the compound. The yellow contours represent regions where steric bulk is not preferred in the compound, i.e., small groups can be attached to these regions. The green contours represent regions where steric bulk is preferred, i.e., attachment of bulky groups will be favorable. The green contour at C20 and S2 indicates that the substitution by bulkier groups will increase the activity of the molecule. The bright yellow contour at C16 and C26 indicates that the substitution by bulkier groups would decrease the activity of the molecule. This contour map gives us some general insight into the nature of the receptor ligandbinding region. The results were predicted, from which one best QSAR result - glucoraphanin (ID 4) was further taken for Molecular dynamics simulation. At least 120 different glucosinolates have been identified. Glucobrassicin and glucoraphanin are generally found in

\section{Table 1: List of non-toxic ligands with docked score}

\begin{tabular}{lll}
\hline Name of the ligand & pIC50 value $(\boldsymbol{\mu m})$ & Docked score \\
\hline Flavanol & 4.677 & 43.59 \\
Glucoiberin & 5.42 & 582.51 \\
Gluconapin & 4.06 & 607.67 \\
Glucoraphanin & 4.55 & 423.74 \\
Neochlorogenic acid & 5.32 & 221.21 \\
Phenylethyl isothiocyanate & 4.59 & 48.77 \\
Progoitrin & 5.95 & 260.29 \\
\hline
\end{tabular}

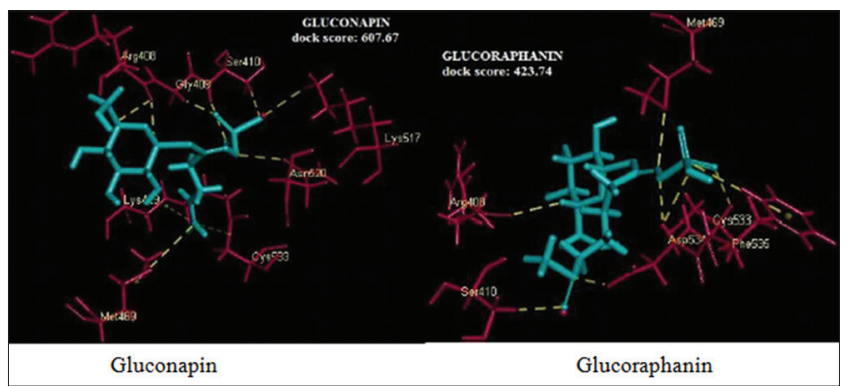

Fig. 1: Docked structures of top scoring ligands 


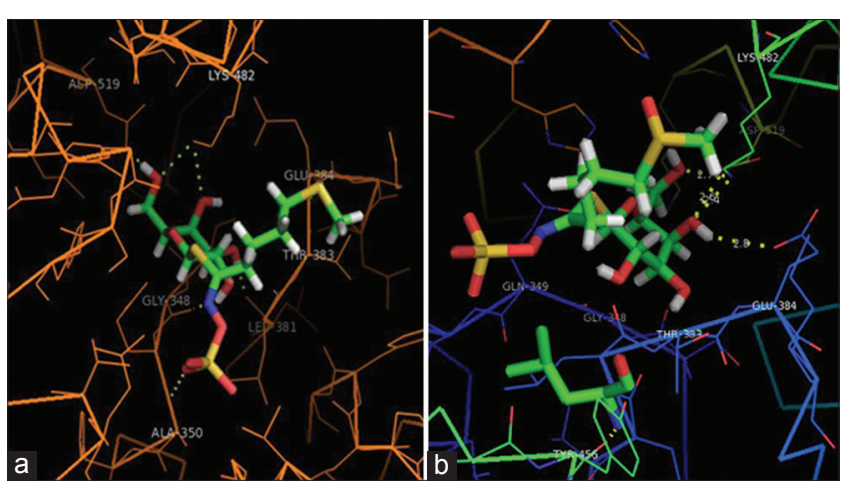

Fig. 2: (a) Interacting residues before simulation. (b) Interacting residues after simulation

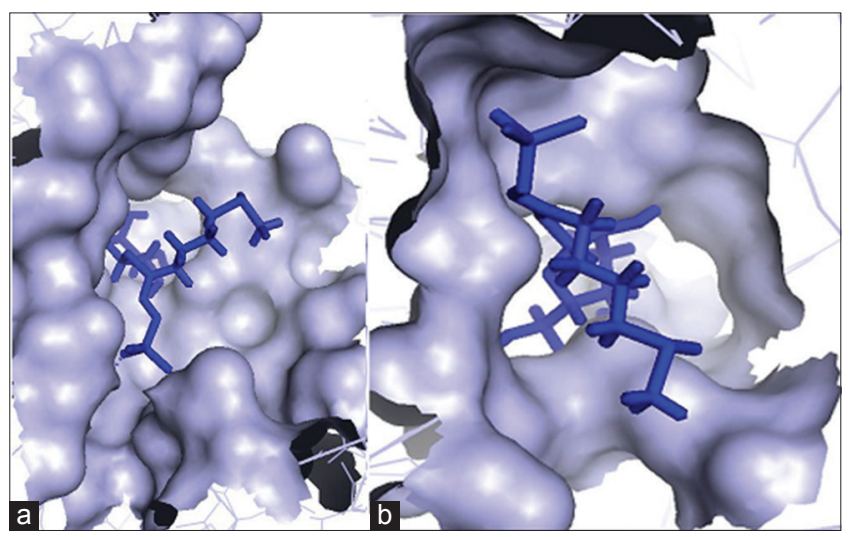

Fig. 3: (a) Presimulated annealing. (b) Postsimulated annealing

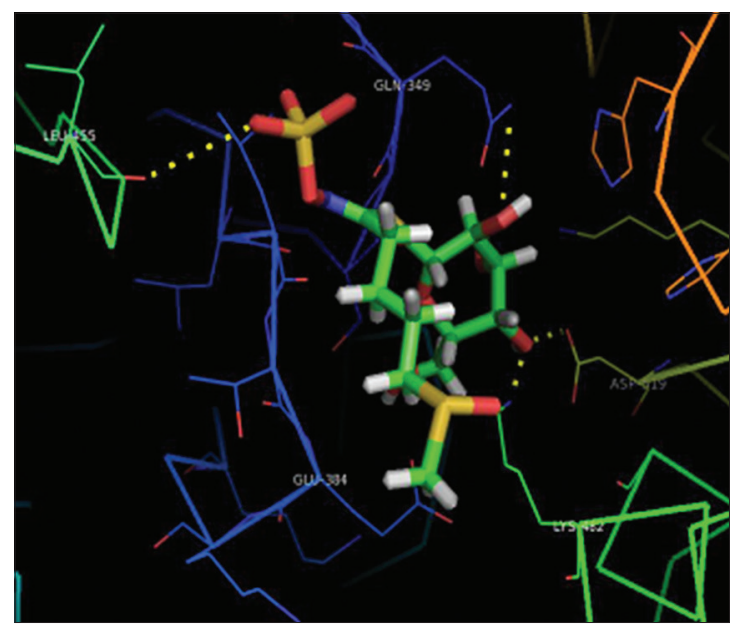

Fig. 4: Interacting residues after simulation annealing

high concentrations in Brassica oleracea, broccoli, Chinese cabbage, radish, and watercress contain high amount of gluconasturtiin whereas sinigrin is found in high concentrations in brussels sprouts, green cabbage, and cauliflower $[16,13]$.

\section{Molecular dynamics simulation}

The model produced after 1000 ns of molecular dynamic simulation shows that the LYS 482 and TYR 456 were located very close to glucoraphanin. Other residues found to interact with glucoraphanin were ASP 519, THR 383, GLU384, SER 347, GLY 348, and GLN 349 as shown in Figs. 3 and 4. Among these residues, LYS482, THR 383, ASP519, and GLU 384 residues interacted with glucoraphanin during the interaction study. Four residues were found to be in contact with glucoraphanin even after simulation. Glucoraphanin was found to satisfy the Lipinski's rule of five. Hence, it can be said that glucoraphanin is likely to have proper absorption and has the drug likeness property.

\section{CONCLUSION}

The interaction studies of the phytochemicals with NF-kB showed considerably good interaction which was observed by the dockscores of the phytochemicals with the target. The interacting residues of glucoraphanin and NF-kB were analyzed. The selection of non-toxic phytochemicals is a major step as the phytochemical when formulated into a drug should be nontoxic to the human body. 3D-QSAR modeling of 7 non-toxic phytochemicals resulted in selection of the best QSAR model of the phytochemicals with the least value of predicted activity. The 3D-QSAR model of glucoraphanin was observed to be the best among the 7 phytochemicals. Molecular dynamics simulation of the interacted complex of NF-kB and glucoraphanin for 1000 ns yielded in a stabilized structure of the complex. Of the 7 interacting residues of the docked complex, 4 were found to be in common with the simulated structure and an additional interaction with TYR456 residue of NF-kB. Simulated annealing performed at a temperature of $500 \mathrm{~K}$ after MD simulation also yielded a stabilized structure of the interacted complex. The stability of NF-KB and glucoraphanin even at 500 K reveals that heating or increase in temperature will not affect the efficacy of the phytochemical if formulated as a drug. The phytochemical glucoraphanin from B. oleracea var. capitata f. rubra (red cabbage) also satisfies all drug likeness properties and can be further optimised as a prominent lead after invivo studies against inflammation.

\section{REFERENCES}

1. Eiró N, Vizoso FJ. Inflammation and cancer. World J Gastrointest Surg 2012;4(3):62-72

2. Lu H, Ouyang W, Huang C. Inflammation, a key event in cancer development. Mol Cancer Res 2006;4(4):221-33.

3. Kadioglu O, Seo EJ. Targeting angiogenesis by phytochemicals. Med Aromat Plants 2013;2(5):1-8

4. Russo M, Spagnuolo C, Tedesco I, Russo GL. Phytochemicals in cancer prevention and therapy: Truth or dare? Toxins (Basel) 2010;2(4):517-51 .

5. Wagner AE, Tershulescen M. Health promoting effects of brassicaderived phytochemical: From chemopreventive and anti-inflammatory activities to epigenetic regulation. Oxid Med Cell Longev 2013;2013:Article ID: 964539, 12.

6. Björkman M, Klingen I, Birch AN, Bones AM, Bruce TJ, Johansen TJ, et al. Phytochemicals of Brassicaceae in plant protection and human health - influences of climate, environment and agronomic practice. Phytochemistry 2011;72(7):538-56.

7. Talalay P, Fahey JW. Phytochemicals from cruciferous plants protect against cancer by modulating carcinogen metabolism. J Nutr 2001;131 11 Suppl:3027S-33.

8. Beecher CW. Cancer preventive properties of varieties of Brassica oleracea: A review. Am J Clin Nutr 1994;59(5):1166-70.

9. Cartea ME, Francisco M, Soengas P, Velasco P. Phenolic compounds in Brassica vegetables. Molecules 2010;16(1):251-80.

10. Kumar S, Andy A. Health promoting bioactive phytochemicals from Brassica. Int Food Res J 2012;19(1):141-52.

11. Gadhe CG, Madhavan T, Kothandan G, Cho SJ. In silico quantitative structure-activity relationship studies on P-gp modulators of tetrahydroisoquinoline-ethyl-phenylamine series. BMC Struct Biol 2011;11:5.

12. Yadav DK, MeenaA, SrivastavaA, ChandaD, KhanF, Chattopadhyay SK. Development of QSAR model for immunomodulatory activity of natural coumarinolignoids. Drug Des Devel Ther 2010;4:173-86.

13. Priyadharshini SE, Ramalingam C, Ramesh B. Designing a novel $\beta$ lactamase inhibitor by using docking and QSAR studies. Int J Pharm Pharm Sci 2014;6(4):516-23.

14. Zhang B, Li Y, Zhang H, Ai C. 3D QSAR and molecular docking studies on derivatives of MK-0457, GSK 1070916 and SNS 314 as inhibitors against aurora B kinase. Int J Mol Sci 2010;11:4326-47.

15. Sharma RB, Gogoi M, Baishya B, Johari S. 3D QSAR and docking studies of a series of Hamamelitannan derivatives as potential PBP4 inhibitors. Int J Pharm Pharm Sci 2014;6(6):558-62

16. Devi JR, Thangam EB. Mechanism of anticancer activity of sulforaphane from Brassica oleracea in HEp-2 human epithelial carcinoma cell line. Asian Pac J Cancer Prev 2012;13(5):2095-100. 